Delft University of Technology

\title{
Phase-Coded FMCW Automotive Radar: System Design and Interference Mitigation
}

Uysal, Faruk

DOI

10.1109/TVT.2019.2953305

Publication date

2020

Document Version

Accepted author manuscript

Published in

IEEE Transactions on Vehicular Technology

\section{Citation (APA)}

Uysal, F. (2020). Phase-Coded FMCW Automotive Radar: System Design and Interference Mitigation. IEEE Transactions on Vehicular Technology, 69(1), 270-281. [8897604].

https://doi.org/10.1109/TVT.2019.2953305

\section{Important note}

To cite this publication, please use the final published version (if applicable).

Please check the document version above.

Other than for strictly personal use, it is not permitted to download, forward or distribute the text or part of it, without the consent of the author(s) and/or copyright holder(s), unless the work is under an open content license such as Creative Commons.

\section{Takedown policy}

Please contact us and provide details if you believe this document breaches copyrights.

We will remove access to the work immediately and investigate your claim. 


\title{
Phase-Coded FMCW Automotive Radar: System Design and Interference Mitigation
}

\author{
Faruk Uysal, Senior Member, IEEE
}

\begin{abstract}
A frequency-modulated continuous-wave (FMCW) radar takes advantage of stretch processing (dechirping, deramping) to reduce the sampling requirements of radar systems such as automotive radars. When applying phase coding into FMCW radar systems for interference mitigation or joint communication, preservation of this benefit is not possible, due to the nature of this process. This paper proposes a new phase coded FMCW system as well as a processing strategy based on a groupdelay filter to deal with unaligned phase-coded radar return signals. Moreover, this paper proposes an improved interference mitigation method based on a phase-coded linear- frequencymodulated (LFM) continuous waveform and points out the improvements in the signal-to-interference ratio (SIR). In the proposed mitigation technique, unlike the traditional approach where RF spectrum spreads, the beat frequency spectrum of the FMCW radar spreads, thus the bandwidth of RF hardware stays the same as a classical LFM continuous wave radar. The success of the proposed phase coded frequency-modulated continuous-wave (PC-FMCW) radar is illustrated in simulations and increased interference mitigation performance is validated by experiments.
\end{abstract}

Index Terms-Phase-Coded Radar, PC-FMCW, interference mitigation, automotive radar.

\section{INTRODUCTION}

$\mathbf{R}$ ECENTLY improvements in self-driving technology emphasize the importance of sensors that are being used in vehicles. Automotive radar -which acts as the digital eyes of self-driving vehicles- is becoming a key element due to its all-weather, day and night capabilities. Achievements in integrated circuits and semiconductors made the low-cost mass production of single-chip automotive radars possible. Most of today's vehicles are already equipped with radar systems to improve road safety. Soon, the coexistence of multiple automotive radars in congested traffic will be an issue with the increasing number of radar-equipped vehicles on the roads. The interference caused by other automobiles negatively affects the functionality of the radar systems by decreasing their detection capability.

A linear frequency modulated (LFM) waveform has been used as the primary waveform in most of the operational radar systems for several decades. However, there is a growing interest in the use of phase coded (PC) waveforms since phase coding can be used to reduce interference between radars in the same vicinity [1]. Radio frequency interference (RFI) is a serious issue for radar applications (such as in automotive radars [2]) due to the crowded electromagnetic spectrum and

Faruk Uysal (f.uysal@tudelft.nl) is with Microwave Sensing, Systems and Signals (MS3) group, at Faculty of Electrical Engineering, Mathematics and Computer Science, Delft University of Technology, Delft, Netherlands. may be observed as synchronous or asynchronous interference [3], which needs to be mitigated for reliable self-driving vehicles.

Many code families have been explored as phase-coded radar waveforms in the past [4], [5] Performance of a code (or a phase coded waveform) is usually measured by the ambiguity function, which jointly demonstrates the propagation delay and Doppler relationship. The Doppler resilient-longer codes are preferred in phase-coded continuous wave (PC-CW) radar applications due to their range sidelobes provided by the code's autocorrelation [6]. There are different architectures for PC-CW radar systems [7]. Such a system may use multiple decoders for each range gate (range channels), which decodes the signal with a shifted version of the code signal. Levanon and Getz compared LFM and PC-CW radars and proposed a multi-correlation processor for PC-CW radar systems [8].

Matched filtering [9], [10] or a sequential search correlation [11] are currently used for the processing of the PC waveforms to reach the optimal Signal-to-Noise ratio (SNR). In literature, mismatched filters are also introduced to lower range sidelobes in PC waveforms [1]. Most of these methods increase either the computational complexity or the complexity of the hardware architecture (such as using multiple correlation lines). On the other hand, using mismatched filters or using correlators in the digital domain requires wideband data acquisition, which can be achieved by a high-end large bandwidth analog-todigital converter (ADC). Thus, these approaches are not suitable for automobile radar applications where the performance to cost ratio is a crucial factor in automobile radar design.

MIMO schemes are applied in automotive radar by using orthogonal waveforms to provide spatial diversity. The initial approach for orthogonal waveforms through code division has been proposed by de Wit et al. for FMCW MIMO (multipleinput, multiple-output) radar [12]. However, the approach is based on frequency domain processing, which can be realizable only in the digital domain, but not in the hardware of a radar system. Kim et al. propose a code division multiplexing method for automotive MIMO radars by selecting the combined frequency shift key-linear FMCW waveform [13]. However, this method, a division of the frequency spectrum for shift keying, yields a decrease in range resolution for each step. In [14], Feger et al. introduce optimized phase codes and weight functions for binary phase coded frequency-modulated continuous-wave (PC-FMCW) MIMO radars by assuming the modulation is narrow-band and by sampling of the intermediate frequency signal at a moderate rate, which means the duration of a single code-chip is much longer than the roundtrip delay time. In some cases, this assumption may not be true 
and the envelope of the received signal needs to be aligned before decoding. The initial system design for a phase-coded linear frequency modulated continuous wave (PC-LFMCW) radar was introduced at [15], [16], without considering the coded beat frequency shifts due to different target delays. As emphasized in the literature, the misalignment of the returned signal is an important issue and this needs to be corrected to achieve the best SNR.

The closest approach to solve the misalignment problem (without using multiple correlation lines) was proposed by Hemmingsen et al. as waveform-diverse stretch processing [17], which proposes replacing the final fast Fourier transform (FFT) stage by a compensation transform that permits the use of other chirp-like waveforms, without changing the RF receive chain. Recently, McCormick et al. use waveform diverse stretch processing [17] to implement an FMCW phaseattached radar/communications system [18], which aims at joint sensing and communication, but not interference mitigation. However, these methods are computationally intense and cannot be applied to a platform where processing power is limited, such as automotive radar systems. In this paper, we address the issue of beat frequency alignment by using a group delay filter which does not only efficiently align the beat frequencies from different targets, but can also be implementable in automotive (or a single-chip) radar systems without increasing the unit cost.

Our proposed method for beat frequency alignment by using group delay filters, can be implemented into any kind of FMCW radar systems. However, in this paper we especially concentrate on a phase-coded LFM waveform on an FMCW radar system and its proper implementation for improved interference mitigation. We propose a new hybrid architecture (hardware and software) for FMCW radar and a signal processing technique to make stretch processing possible, even when different time delays are present between the transmitted and received phase coded waveforms In other words, our method addresses the alignment issue of the beat frequencies without increasing the unit cost and computational complexity. We extend the proposed hybrid approach to a hardware based system architecture for PC-FMCW radar. The phase-coded linear frequency modulated continuous wave (PC-FMCW) radar architecture with beat frequency alignment feature is novel and has, to the best of the authors' knowledge, not been presented in literature before. Moreover, for the first time, the proposed methods are successfully applied to interference mitigation problems in radar systems.

The remainder of this paper is structured as follows. Section II explains the system model of a traditional FMCW radar system, as well as the phase coding. Then, the proposed system is introduced in Section III including envelope alignment through a group delay filter and decoding procedures. The proof of concept is illustrated by simulations and experiments in Section IV. The application of the proposed method for interference mitigation on an LFMCW radar is discussed in Section V The hardware implementation of the proposed method and future works are discussed in Section VI. Finally, Section VII presents the conclusions.

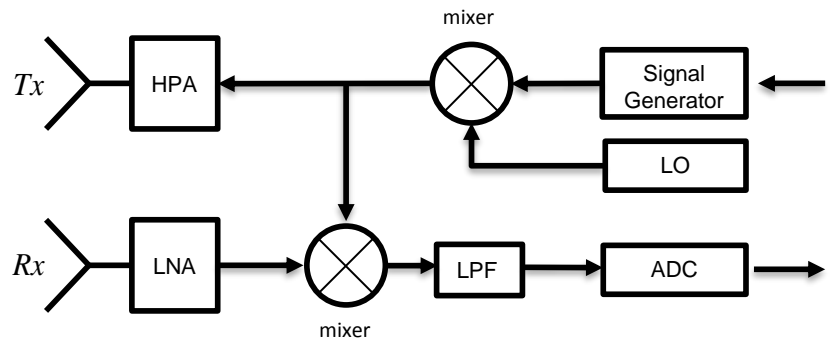

Fig. 1. Simplified block diagram of a traditional FMCW radar system.

\section{SYSTEM MODEL}

\section{A. Background}

In frequency modulated continuous wave (FMCW) radar, the frequency of the transmit signal changes over time, usually in the form of a linear sweep over a predetermined bandwidth. The frequency difference between the transmitted and received signals is determined by mixing these two signals, and can be used to measure the distance and velocity of the objects [19]. A simplified block diagram of a traditional FMCW radar is illustrated in Figure 1.

Let us assume a signal generator, such as an arbitrary waveform generator (AWG) or a voltage controlled oscillator (VCO) or a direct digital synthesis (DDS) [20], creates a waveform whose instantaneous frequency is shown in Figure 2.a (where the duration of different possible events is marked as follows: $T_{d w e l l}$ is the dwell time; $T_{\text {settle }}$ is the settling time of the waveform generator; $T_{A D C}$ is the ADC sampling time; $T_{\text {reset }}$ is the reset time of the waveform generator).

The instantaneous frequency of the waveform is set by $f_{i}(t)=k t$, where the slope of the waveform $k=B / T$ is equal to the ratio of bandwidth $B$, and the duration $T=T_{\text {settle }}+T_{A D C}$ of the waveform. Then, the instantaneous phase of the radio-frequency (RF) waveform with an initial phase $\phi_{0}$ is defined as,

$$
\begin{aligned}
\phi(t) & =2 \pi \int_{0}^{t} f(t) d t \\
& =2 \pi\left(f_{c} t+\frac{1}{2} k t^{2}\right)+\phi_{0} .
\end{aligned}
$$

A transmit waveform with an envelope $A(t)$ can be written as

$$
x_{T}(t)=A(t) \operatorname{rect}\left(\frac{t-T / 2}{T}\right) \cos (\phi(t)),
$$

where a rect function (for a pulse centered at $X$ and has width $Y$ ) can be written in the form:

$$
\operatorname{rect}\left(\frac{t-X}{Y}\right) \text {. }
$$

The received waveform which is reflected from a target at a distance $R_{0}$ with a line of sight velocity $\nu$ can be written as a delayed copy of the transmitted signal (for simplicity of the derivation assuming no free-space path loss),

$$
x_{R}(t)=A(t-\tau) \operatorname{rect}\left(\frac{t-\tau-T / 2}{T}\right) \cos (\phi(t-\tau))
$$


where the round-trip time delay is

$$
\tau=\frac{2\left(R_{0}+\nu t\right)}{c}
$$

The received and transmitted signals are mixed in order to reduce the required sampling rate. The mixing generates the intermediate-frequency (which is also known as beat, dechirped, deramped ) signal is

$$
\begin{aligned}
x_{B}(t) & =x_{T}(t) x_{R}(t) \\
& =A(t) A(t-\tau) \operatorname{rect}\left(\frac{t-\tau-T / 2}{T}\right) \\
& \cos (\phi(t)) \cos (\phi(t-\tau))
\end{aligned}
$$

which can be also written as,

$$
\begin{array}{r}
x_{B}(t)=\frac{A(t) A(t-\tau)}{2} \operatorname{rect}\left(\frac{t-\tau-T / 2}{T}\right) \\
(\cos (\phi(t)-\phi(t-\tau))+\cos (\phi(t)+\phi(t-\tau))) .
\end{array}
$$

The beat signal is then low-pass filtered to eliminate the high frequency (sum) components before being digitized by an $\mathrm{ADC}$ at a rate of at least twice the maximum beat frequency

a)

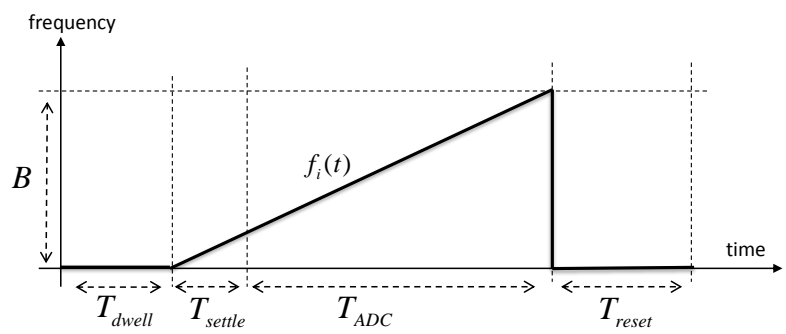

b)

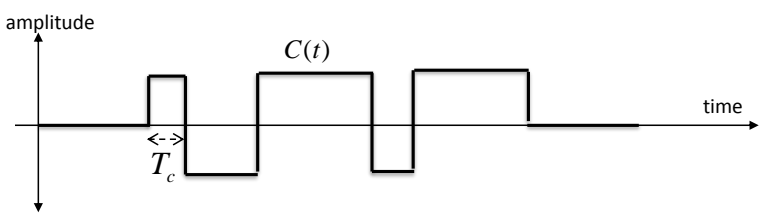

c)

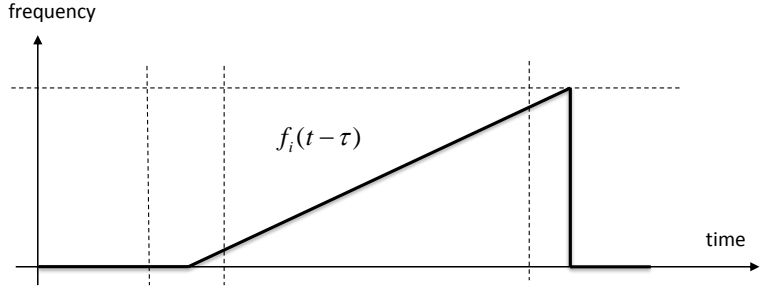

d)

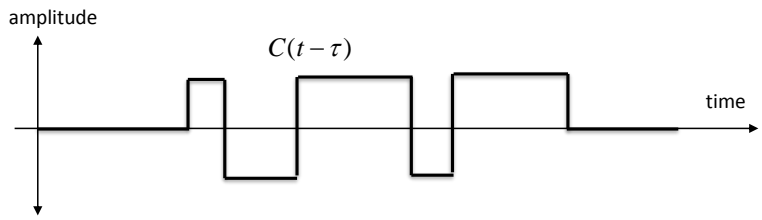

Fig. 2. Illustration of typical transmitted and received signal with their envelopes. a) The instantaneous frequency of the transmitted signal. b) The envelope of transmitted signal as bipolar binary coding. c) The instantaneous frequency of the received signal. b) The envelope of the received signal as bipolar binary coding. $f_{b, \max }$. Only the low-frequency (difference) components pass out of the low-pass filter, which yields a beat signal

$$
\begin{aligned}
& x_{B}(t)=\frac{A(t) A(t-\tau)}{2} \operatorname{rect}\left(\frac{t-\tau-T / 2}{T}\right) \\
& \cos (\phi(t)-\phi(t-\tau)) .
\end{aligned}
$$

\section{B. Phase Coding}

Phase coded waveforms usually divide the pulse into $L_{c}$ time segments. Each time segment, known as a chip, may have a different phase than the others. The chip phase can be 0 or $\pi$ for binary phase codes, while polyphase codes support more phase changes [1]. A single period of a phase-coded waveform for a binary sequence $\alpha_{1}, \alpha_{2}, \ldots, \alpha_{L_{c}}$ for $L_{c}$ bits $\alpha_{n} \in\{-1,+1\}$, can mathematically be represented as,

$$
C(t)=\sum_{n=1}^{L_{c}} e^{j \phi_{n}} \operatorname{rect}\left(\frac{t-(n-1 / 2) T_{c}}{T_{c}}\right),
$$

where $\phi(n) \in\{0, \pi\}$ denotes the phase corresponding to the $n^{\text {th }}$ bit of the sequence. The phase is kept constant for a chip duration $T_{c}=T / L_{c}$. As the concurrent chip value changes from -1 to +1 , or vice versa, a phase discontinuity will take place as the phase is shifted $180^{\circ}$. If the transmit waveform is coded with a bipolar phase code $C(t) \in\{-1,1\}$, then the envelopes of the transmitted and received signals become $A(t)=C(t)$ and, $A(t-\tau)=C(t-\tau)$, respectively. The beat signal for the phase-coded FMCW radar after dechirping and low-pass filtering can be written as,

$$
\begin{aligned}
x_{B}(t)=\frac{C(t) C(t-\tau)}{2} \operatorname{rect}( & \left.\frac{t-\tau-T / 2}{T}\right) \\
& \cos (\phi(t)-\phi(t-\tau)) .
\end{aligned}
$$

As illustrated Figure 2.b and Figure 2.d, the transmitted $C(t)$ and the received $C(t-\tau)$ (assuming no loss) signal envelopes do not match (in time) with each other due to the roundtrip time delay. In that case, it is not possible to successfully decode the received signal. Thus, stretch processing does not give expected range profiles after spectral analysis. A new processing theme is necessary to align the transmitted and the received signals' envelopes for perfect decoding, which is discussed in the following section.

\section{PROPOSED SySTEM MODEL}

The proposed phase coded FMCW radar system (which is illustrated in Figure 3) has two distinctive features compared to a traditional FMCW radar system.

Firstly, the received signal is mixed with the uncoded transmit signal which preserves original phase coding for each beat frequency after dechirping. Thus, for the proposed phase-coded FMCW radar architecture, the beat signal after dechirping and low-pass filtering can be written as,

$$
\begin{aligned}
& x_{B}(t)=\frac{C(t-\tau)}{2} \operatorname{rect}\left(\frac{t-\tau-T / 2}{T}\right) \\
& \cos (\phi(t)-\phi(t-\tau)) .
\end{aligned}
$$




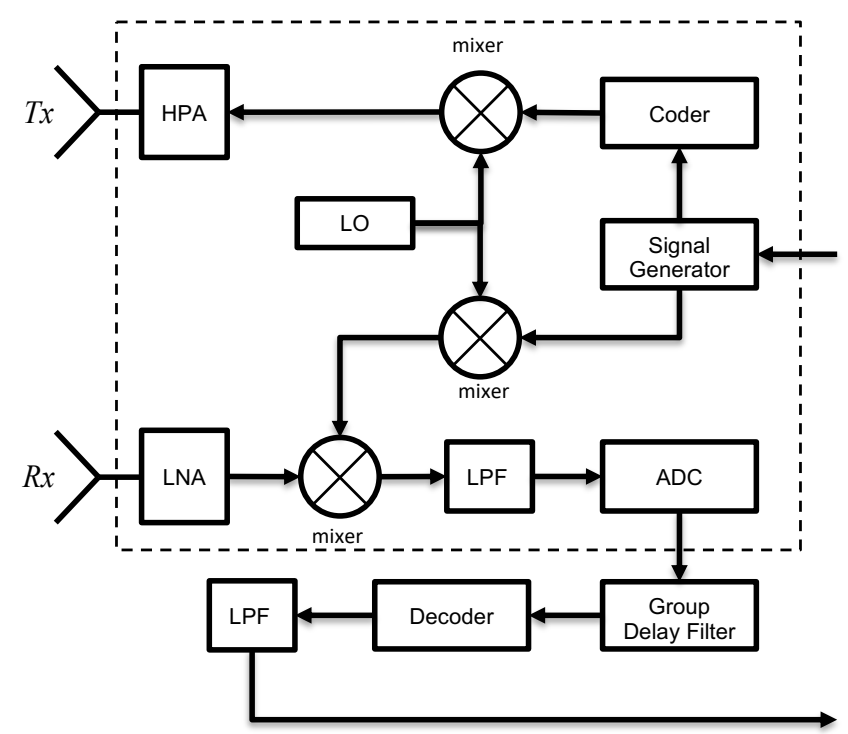

Fig. 3. A simplified block diagram of a proposed Phase-Coded FMCW radar system. The radar transceiver's part, with phase coding capabilities, is highlighted by a dashed line. (For instance, such a transceiver may have binary phase shift keying (BPSK) capabilities [21].)

Secondly and more importantly, the proposed system uses a simple time-domain group delay filter (which can also be implemented in the frequency domain equivalently) to align the envelope of each beat frequency which makes it possible to successfully decode the received signal, even though each beat frequency is delayed differently in time.

\section{A. Envelope Alignment}

Figure 4.a illustrates the beat frequencies in the timefrequency domain without any processing. The beat frequen-

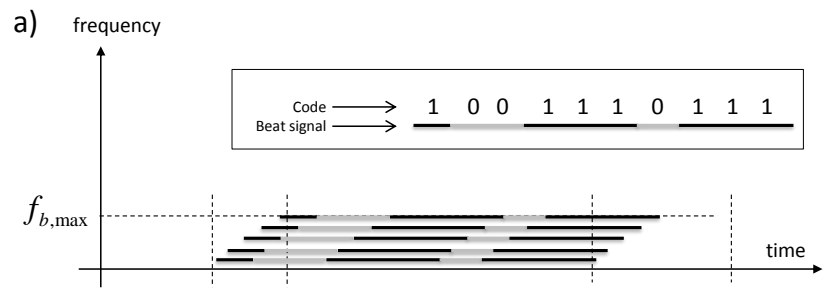

b) frequency
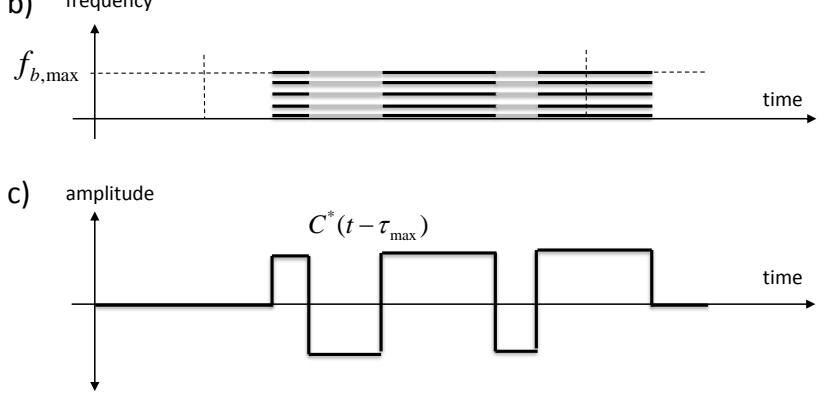

Fig. 4. Illustration of a) beat frequencies without any processing;b) beat frequencies after a group delay filter; c) delayed bipolar binary decoding signal. cies that belong to the far range targets are delayed more than the beat frequencies that belong to the close range targets. Thus, each beat frequency is shifted differently than the others.

The exact time delay of a beat frequency for an arbitrary range $R$ is written as,

$$
\tau_{b}=\frac{2 R}{c},
$$

whereas a beat frequency is [22]

$$
f_{b}=\frac{2 R}{c} \frac{B}{T} .
$$

Alternatively, combining (12) and (13), we find that a beat frequency $f_{b}$ was delayed by

$$
\tau_{b}=\frac{T}{B} f_{b}=\frac{f_{b}}{k} .
$$

Note that, the maximum beat frequency $f_{b, \max }=f_{s} / 2$ is delayed with the maximum delay $\tau_{\max }$ due to the nature of round trip delay from maximum range $R_{\max }$. To align the envelope of all beat frequencies, each beat frequency needs to be delayed to the same time instance with $f_{b, \max }$. Thus, all beat frequencies $0<f_{b}<f_{b, \max }$ need to be delayed with

$$
\begin{aligned}
\tau_{D} & =\tau_{\max }-\tau_{b} \\
& =\frac{1}{k}\left(f_{b, \max }-f_{b}\right) .
\end{aligned}
$$

\section{B. Group Delay Filter}

In reality, a received signal $x(t)$ consists of multiple beat frequencies, since echoes are received simultaneously from multiple targets. For $P$ targets at arbitrary ranges, the overall received signal can be written as

$$
x(t)=\sum_{p=1}^{P} x_{B}\left(t, \tau_{p}\right),
$$

where $\tau_{p}$ is the time delay for $p^{\text {th }}$ target. Note that for the proposed system in Figure 3, the received signal is dechirped with the uncoded waveform $A(t)=1$. Thus the beat signal for the proposed method is written as in (11).

To tackle with the shifted received signal envelopes, explicitly $C\left(t-\tau_{p}\right)$, we propose to use a time-domain group delay filter which delays all frequency components of the input signal independently. A group-delay filter is an all-pass filter that passes all signals, but the delay for signal components will be different for the various frequencies. Thus, it is an ideal candidate to implement the proposed delay at (15).

Note that the group delay of a filter is defined as the negative derivative of the phase. For a filter with a magnitude response $|H(f)|$ and a phase response $\angle H(f)$, explicitly

$$
H(f)=|H(f)| \angle H(f),
$$

its group delay is defined as

$$
\tau_{G D}(f)=-\frac{1}{2 \pi} \frac{d}{d f} \angle H(f) .
$$


For an arbitrary signal in the form of $y(t)=E(t) \cos (\phi(t))$, where $E(t)$ is the slow varying envelope, the output of the group delay filter $h(t)$ is defined as [23],

$$
z(t)=y(t) \otimes h(t) \approx E\left(t-\tau_{G D}(f)\right) \cos \left(\phi\left(t-\tau_{\phi}(f)\right)\right)
$$

Here, the envelope function is delayed by the group delay, whereas the carrier (in our case the beat signal) is delayed by the phase delay which is

$$
\tau_{\phi}(f)=-\frac{1}{2 \pi} \frac{\angle H(f)}{f} .
$$

Let us define a filter $h_{G D}$ with a constant magnitude response $|H(f)|=1$ and a group-delay $\tau_{G D}=\tau_{D}$ which yields a phase response

$$
\begin{aligned}
\angle H(f) & =-2 \pi \int \tau_{G D}(f) d f \\
& =-2 \pi \int \frac{1}{k}\left(f_{b, \max }-f_{b}\right) d f_{b} \\
\angle H\left(f_{b}\right) & =\frac{2 \pi f_{b}}{k}\left(\frac{f_{b}}{2}-f_{b, \max }\right) .
\end{aligned}
$$

Realization of such a digital filter, for given magnitude and phase responses, is possible by least $p^{\text {th }}$-norm iterative optimization [24].

As a result of this filter, the envelope of the received signal is delayed as desired for all frequency components (beat frequencies). Note that there will be a phase delay for each beat frequency, as seen from the second part of (19), explicitly $\cos \left(\phi\left(t-\tau_{\phi}(f)\right)\right)$. In practice, we use spectral estimation methods (such as fast Fourier transform, FFT) to exploit the range information after stretch processing. Since the frequency component is exploited by spectral analysis, a phase delay (change) in the beat signal does not affect the range estimates. The time-domain output signal after group delay filtering can be written as

$$
\begin{aligned}
\tilde{x}(t) & =x(t) \otimes h_{G D}(t) \\
& =\sum_{p=1}^{P} \frac{C\left(t-\tau_{\max }\right)}{2} \operatorname{rect}\left(\frac{t-\tau_{\max }-T / 2}{T}\right) \\
& \cos \left(\phi\left(t-\tau_{\phi}\right)-\phi\left(t-\tau_{p}-\tau_{\phi}\right)\right) .
\end{aligned}
$$

Even though a single group delay filter, $h_{G D}$, is applied in the time domain, the output of the filter, $\tilde{x}(t)$, is illustrated in the time-frequency domain to demonstrate the influence of the filter on beat frequencies. Figure 4.b shows the beat frequencies after the proposed group-delay filter. As illustrated in the figure, all beat frequencies are aligned with the $f_{b, \max }$ at the same time instance $\tau_{\max }$.

\section{Decoding}

At the beginning of this section, we emphasised that one of the important features of the proposed architecture is to preserve phase codes in time-domain received signal. Decoding needs to be applied just before the spectral estimation to achieve correct range information. Without decoding, spectral estimation techniques can not exploit the frequency content, due to the abrupt phase changes in the beat signal, thus range information can not be achieved.

It should be noted that, after the group-delay filtering, all beat frequencies are aligned at the maximum delay as seen in Figure 4.b. A proper decoding signal should correct the phase changes initiated by the coding signal $C(t)$, so the decoding signal should be the complex conjugate ${ }^{1}$ of the coding signal (in our case the coding signal itself can be used, due to the binary coding). Moreover, the decoding signal should be perfectly aligned with the received signal. Under these circumstances, a proper decoding signal can be defined as $C^{*}\left(t-\tau_{\max }\right)$, which is illustrated in Figure 4.c for this specific example. Finally, the decoded beat signal is given by

$$
\begin{aligned}
s(t)= & C^{*}\left(t-\tau_{\max }\right) \tilde{x}(t) \\
= & \frac{1}{2} \sum_{p=1}^{P} \operatorname{rect}\left(\frac{t-\tau_{\max }-T / 2}{T}\right) \\
& \quad \cos \left(\phi\left(t-\tau_{\phi}\right)-\phi\left(t-\tau_{p}-\tau_{\phi}\right)\right),
\end{aligned}
$$

since $C\left(t-\tau_{\max }\right) C^{*}\left(t-\tau_{\max }\right)=u\left(t-t_{\max }\right)$ where

$$
u\left(t-t_{o}\right)= \begin{cases}0 & t<t_{o} \\ 1 & t>t_{o}\end{cases}
$$

is the unit step function.

From now on, traditional FMCW processing techniques can be used to process decoded signal $s(t)$. Bearing in mind that each burst (pulse) (that is filtered by same group-delay filter) has the same amount of phase delay as shown in (20). Thus, Doppler processing is not affected by the proposed algorithm. One may apply a 2D-FFT to a series of decoded signals to achieve the range-Doppler information of the targets.

\section{Demonstration of Concept}

The success of the proposed phase-coded FMCW radar is first demonstrated by simulations. After this, the appropriate signal processing approach is verified by experiments.

\section{A. Simulations}

Even though the proposed method is more general and applicable to different systems, for simulation the radar design parameters are selected similar to a $79 \mathrm{GHz}$ automotive radar system, as summarized in Table I.

TABLE I

Some of the Radar System Design Parameters for Simulation

\begin{tabular}{|lcr|}
\hline ADC Sampling Time & $T_{A D C}$ & $25.60 \mu \mathrm{s}$ \\
\hline Dwell Time & $T_{\text {dwell }}$ & $1.00 \mu \mathrm{s}$ \\
\hline Reset Time & $T_{\text {reset }}$ & $5.00 \mu \mathrm{s}$ \\
\hline Settle Time & $T_{\text {settle }}$ & $3.52 \mu \mathrm{s}$ \\
\hline ADC Sampling Frequency & $f_{s}$ & $40 \mathrm{MHz}$ \\
\hline Bandwidth & $B$ & $2 \mathrm{GHz}$ \\
\hline Number of Chirps & & 512 \\
\hline Number of Bits (Chips) per Chirp & & 16 \\
\hline Duration of Chips & & $1.60 \mu \mathrm{s}$ \\
\hline
\end{tabular}

$\otimes$ is the convolution operator.
${ }^{1}(\bullet)^{*}$ is the complex conjugate of a complex signal. 
1) Coding Matrix: As a coding signal, in this first example we choose a 16-bit Kasami sequence with a generator polynomial $g(z)=\left[z^{6}+z+1\right]$, since Kasami sequences are known to be a sets of sequences that have good cross-correlation properties [25]. Then, a selected code signal is randomly shifted for each pulse (burst). In a coherent processing interval (CPI), 512 bursts (pulses or chips) are transmitted. Thus, a range-Doppler processing frame consists of 512 bursts (slow time samples) and 1024 ADC samples (fast-time samples). Figure 5 illustrates a coding matrix for a range-Doppler processing frame whose rows consist of randomly shifted Kasami codes. Explicitly, each row of the coding matrix is created by applying a random circular shift ${ }^{2}$ to a selected 16bit Kasami sequence. The coding matrix is not unique and can be adapted based on the intended application. For instance, for joint sensing and communication, a coding matrix may carry communication information.

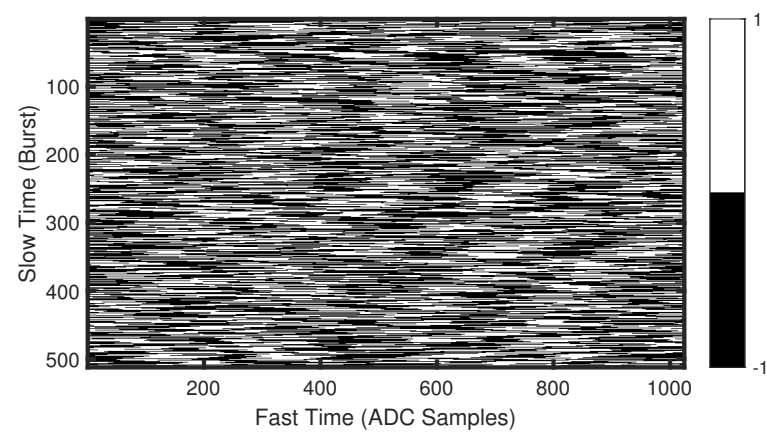

Fig. 5. A coding matrix for a range-Doppler processing frame.

Firstly, a single target, at a range of $10 \mathrm{~m}$ with a velocity of $10 \mathrm{~m} / \mathrm{s}$, is simulated. Figure 6 .a shows a part (155 samples over 1024) of the filter's input signal (unprocessed beat frequency) together with the coding signal. As seen from the figure, due to the round trip delay, the received beat frequency is shifted and its phase change does not coincide with the transmitted code signal. This misalignment can easily be corrected by a single time-domain group delay filter for all beat frequencies (Figure 6 shows a single beat frequency for illustration purpose).

2) Group Delay Filter: A real, stable, infinite impulse response (IIR) group-delay filter is designed (as described in Section III-B), due to its non-constant group delay properties (since a finite impulse response filter has a constant group delay and the proposed method can not be realized) and easy implementations to the radar processors, such as a microcontroller (MC). The filter order is set 30 , which yields a direct-form II filter structure (can be implemented with 60 multipliers and 60 adders). The frequency response of the filter is illustrated in Figure 7. As seen from the figure, even though the requirement for the magnitude response $|H(f)|=1$ is almost satisfied after optimization, there is still a small ripple in the magnitude response.

\footnotetext{
${ }^{2} \mathrm{~A}$ circular shift is the operation of rearranging the entries in a sequence by moving the final entry to the first position, while shifting all other entries to the next position.
}
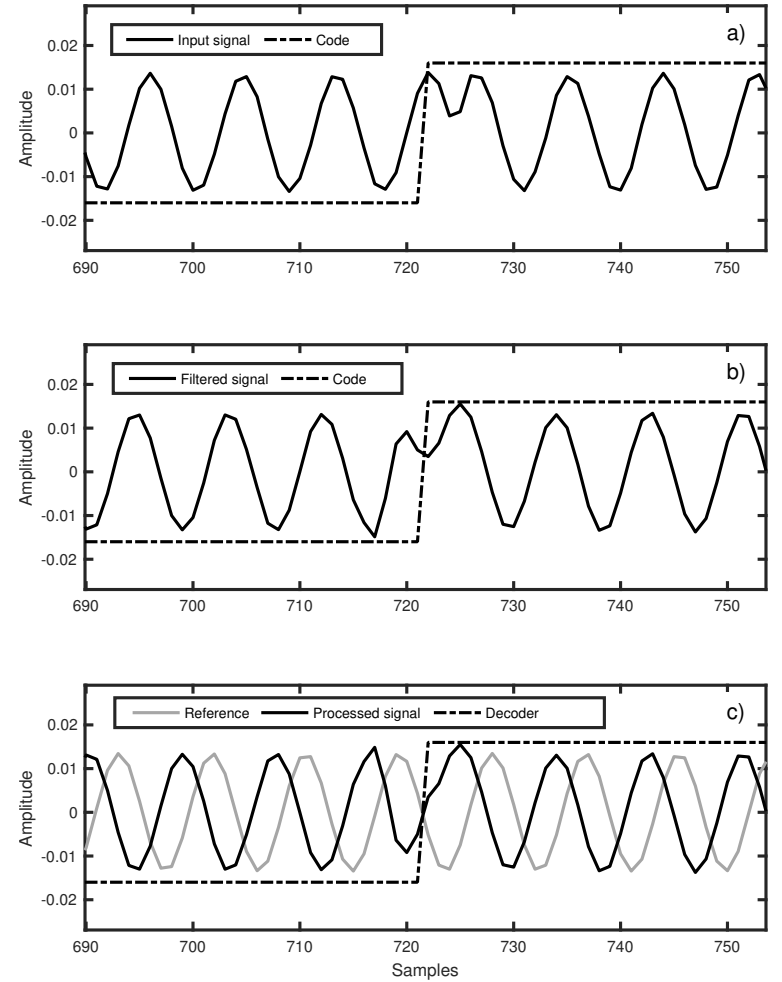

Fig. 6. Illustration of a small part of a) filter input signal (beat frequency), b) filter output, and c) decoded signal after filtering.
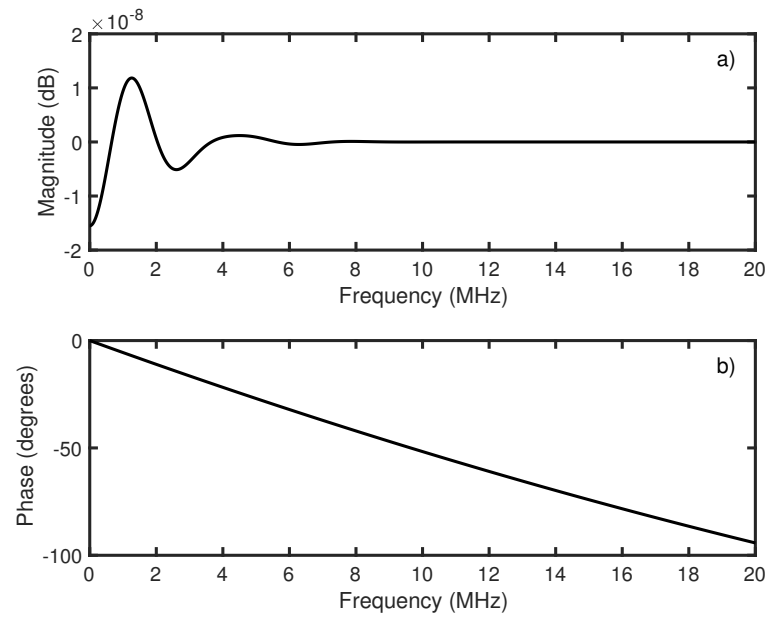

Fig. 7. Bode plot of the designed filter. a) Magnitude response, b) phase response

Figure 8 shows the ideal and designed group delay of the designed IIR filter. The filter itself has a constant delay which is 24.13 samples, as seen from the figure. The comparison of the group delay of the designed filter and the ideal group delay is illustrated in Figure 8. Note that the ideal group delay is shifted by 24.13 samples for easy comparison. Figure 8 also shows the simulated target's beat frequency and delay (34.45 samples). In this example case, the maximum delay is 35.78 samples. Whereas, the delay due to the round trip is $35.78-34.45=1.33$ samples, which can be observed easily from Figure 6.a, since the frequency change occurs 2 samples later than the phase change of the coding signal. 


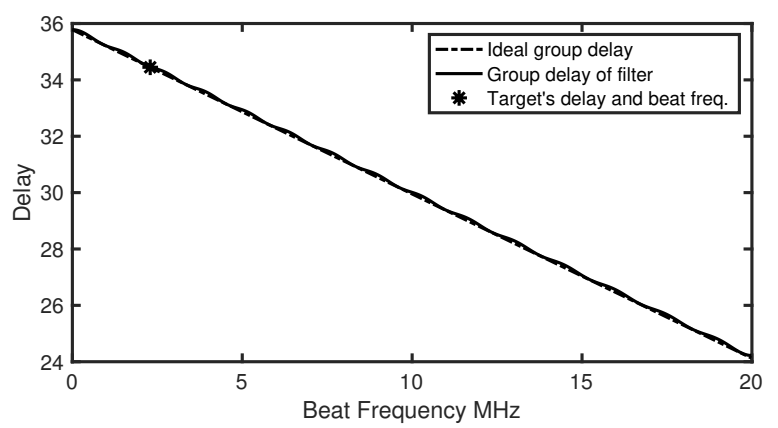

Fig. 8. Group delay of the designed filter.

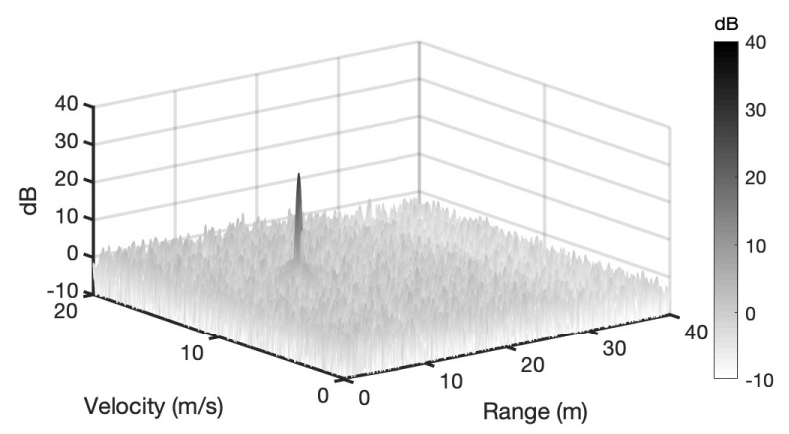

Fig. 9. Range-Doppler plot of the simulated single target with a speed of $10 \mathrm{~m} / \mathrm{s}$ at range $10 \mathrm{~m}$.

The output of the designed filter is illustrated in Figure 6.b, together with the transmitted code signal. It is obvious that the group delay filter managed to align the shifted beat frequency (even though there are small errors due to discretization). Bearing in mind that the filtered signal must be aligned with the maximum delay $\tau_{\max }$, as explained in Section III-A, for illustration purpose, we manually shift the signal back to its original position for easy comparison (phase codes are used as reference points). Note that in real-time processing, this alignment is not needed. Finally, the decoded signal is illustrated in Figure 6.c, together with the overlaid decoder signal as well as a beat signal from a traditional FMCW radar as a reference. The processed signal has a smoother phase compared to the input signal and has been well aligned with the decoder signal. Compared to the reference signal, a phase shift is observed in the processed (output) signal which is expected due to (19).

3) Range Doppler Processing: After filtering and decoding, the beat signals can be processed traditionally. Range-Doppler processing can efficiently be executed by a 2D-FFT. During range-Doppler processing, a Hamming window, in both range and Doppler dimension, is used for sidelobe suppression.

Figure 9 shows the final range-Doppler image of the system, where the simulated target forms a peak at the correct range and velocity. To further investigate the system response, a zero-Doppler (velocity) cut of the range-Doppler output is illustrated in Figure 10. As seen from the figure, the waveform is slightly affected due to a Doppler shift (movement of the target). The detailed investigation of Doppler influence on waveform coding is investigated in [26].

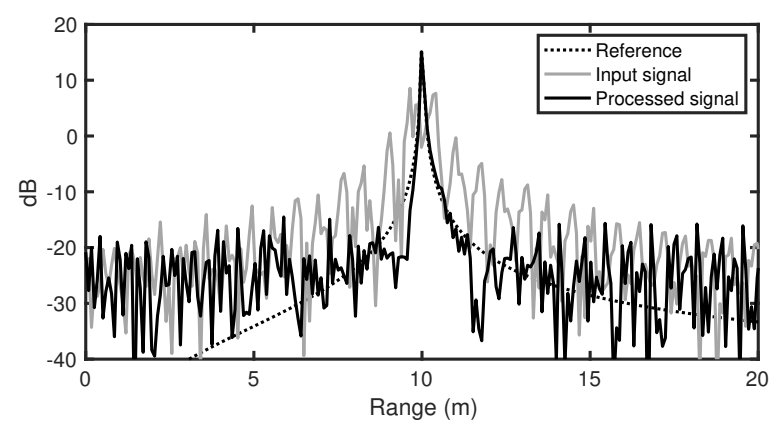

Fig. 10. Range plot of the simulated single target (before Doppler processing). Comparison of traditional FMCW output, unprocessed signal output, and processed (filtered and decoded) signal output

Furthermore, to demonstrate the added value of the proposed filtering approach, the results are compared with the regular stretch processing after phase decoding. Figure 11 illustrates two examples, where the dotted line is used for reference result, which is the output of a traditional FMCW radar system; the gray line is used for the output of stretch processing after phase decoding in a PC-FMCW system; and the black line is used for the result of the proposed filtering, followed with decoding and stretch processing in a PC-FMCW system. As seen in Figure 11.a, the distortion due to the phase coding is not dominant for the closest targets. On the other hand, for targets at the farther range, higher sidelobes are observed due to phase coding, thus the proposed filter is needed before phase decoding, to mitigate these higher sidelobes as illustrated in Figure 11.b. It should be noted that it is challenging to generalize the results. Since some of the standard metrics (e.g. peak to side-lobe ratio) are affected by the selected code family, length of the code and the distribution
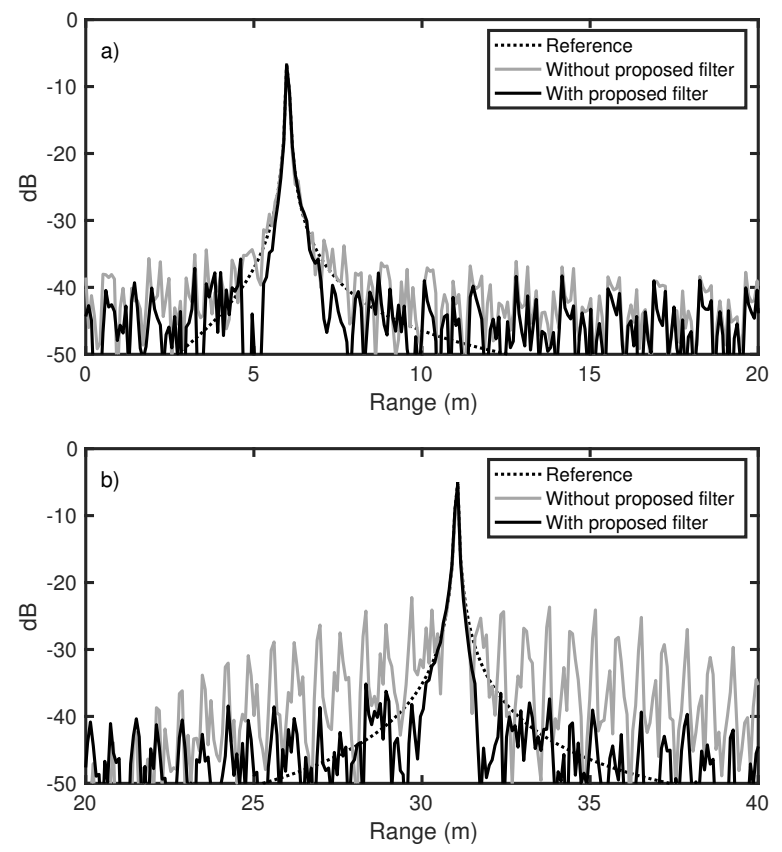

Fig. 11. Improvement of the peak to side-lobe ratio (PSLR) by proposed filtering approach for targets at different ranges: a) stationary target at $6 \mathrm{~m}, \mathrm{~b}$ ) stationary target at $31 \mathrm{~m}$. If not corrected, phase coding increases PSLR. 
and range of targets in the scene. Nevertheless, the proposed filtering approach always gives a better (or in an ideal case gives the best) PSLR, when comparing it to the traditional decoding as illustrated in Figure 11.

\section{B. Multiple Targets}

We extend the simulation to a multiple target scenario to demonstrate the success of the proposed approach, even when there are multiple targets in the same range and/or same velocity (Doppler frequency). A radar path-loss model is included to simulate different signal-to-noise ratio (SNR) levels [27]. The location of five different targets, together with their velocities, radar cross sections (RCS) and SNR values, are summarized in Table II.

Figure 12 shows the result of the aforementioned multitarget scenario. As seen from the figure, all five targets are focused by range-Doppler processing, after group-delay filtering and decoding. It should be noted that a single group delay filter aligns all targets' beat frequencies. Thus a decoding signal can be applied successfully, which yields a well focused range-Doppler output.

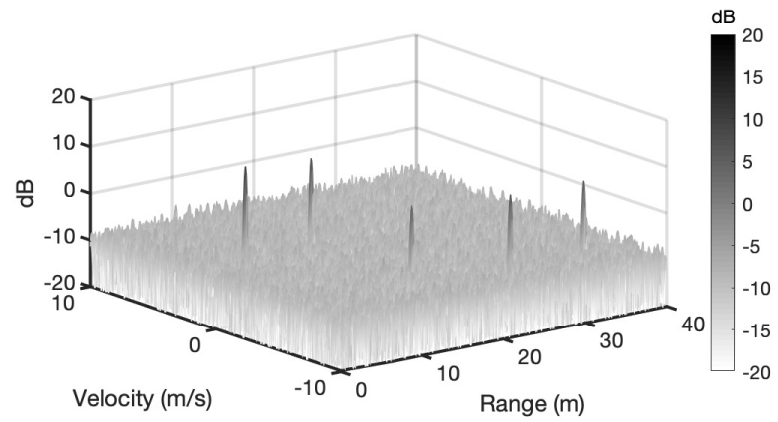

Fig. 12. Range-Doppler plot of multiple targets. Two targets are at the same range and two targets are at the same Doppler.

\section{Experiments}

To validate the results obtained from the simulation model, experiments are set up in an intermediate frequency (IF). Due to the unavailability of a phase-coded FMCW radar, controlled experiments are performed using an arbitrary waveform generator (AWG) and an oscilloscope.

Signal generation for the phase coded-LFM waveform is done in the digital domain using the MATLAB environment. Like with the simulations, 16-bits Kasami sequence is used for phase coding. The sampling frequency for waveform generation is equal to the maximum sampling rate of the AWG

TABLE II

TARGET'S PARAMETERS FOR MULTI TARGET SIMULATION

\begin{tabular}{|c|c|c|c|c|}
\cline { 2 - 5 } \multicolumn{1}{c|}{} & Range $(\mathrm{m})$ & Velocity $(\mathrm{m} / \mathrm{s})$ & RCS $\left(\mathrm{dB} \mathrm{m}^{2}\right)$ & SNR $(\mathrm{dB})$ \\
\hline Target-1 & 6.8 & 2 & 15 & 1.22 \\
\hline Target-2 & 16.4 & -5 & 20 & -8.97 \\
\hline Target-3 & 16.4 & 3 & 25 & -4.04 \\
\hline Target-4 & 25.4 & -7 & 30 & -6.57 \\
\hline Target-5 & 34.3 & -7 & 35 & -6.80 \\
\hline
\end{tabular}

(Tektronix AWG5014B), which equals $1.2 \mathrm{GSa} / \mathrm{s}$. Since the maximum allowable bandwidth is $0.5 \mathrm{GHz}$, the bandwidth of the waveform is selected as $0.4 \mathrm{GHz}$ which yields a range resolution of $0.37 \mathrm{~cm}$. Other parameters are selected as shown in Table I. The AWG is programmed to create a set of delayed signals to mimic target returns, which are collected by an oscilloscope (Agilent DSO-X-91604A) with a speed of $1 \mathrm{GSa} / \mathrm{s}$. Collected waveforms are dechirped and downsampled to $40 \mathrm{MHz}$ to create the beat signals, in a similar way to the simulations.

One of the collected signals is illustrated in Figure 13.a together with the 16 bits coding signal. Unlike the simulation, it is hard to observe the phase changes since there are two targets in the collected data and the amplitude of the collected signal is decreasing towards the high frequencies, due to the internal low-pass filter of the AWG.

A new group delay filter is designed according to (15) to process the experimental data, since the bandwidth of the signal used in the experiments differs from the bandwidth in the simulations. The output of the IIR group delay filter of length 46, is illustrated in Figure 13.b, together with the transmitted code signal. The filtered signal is then decoded and illustrated in Figure 13.c, together with the overlaid decoder signal.

After filtering for envelope alignment and decoding, rangeDoppler processing is applied to a frame which consists of 512 slow-time and 1024 fast-time samples. A Hamming window is applied for both range and Doppler dimension to control the excessive sidelobes. The result of the range-
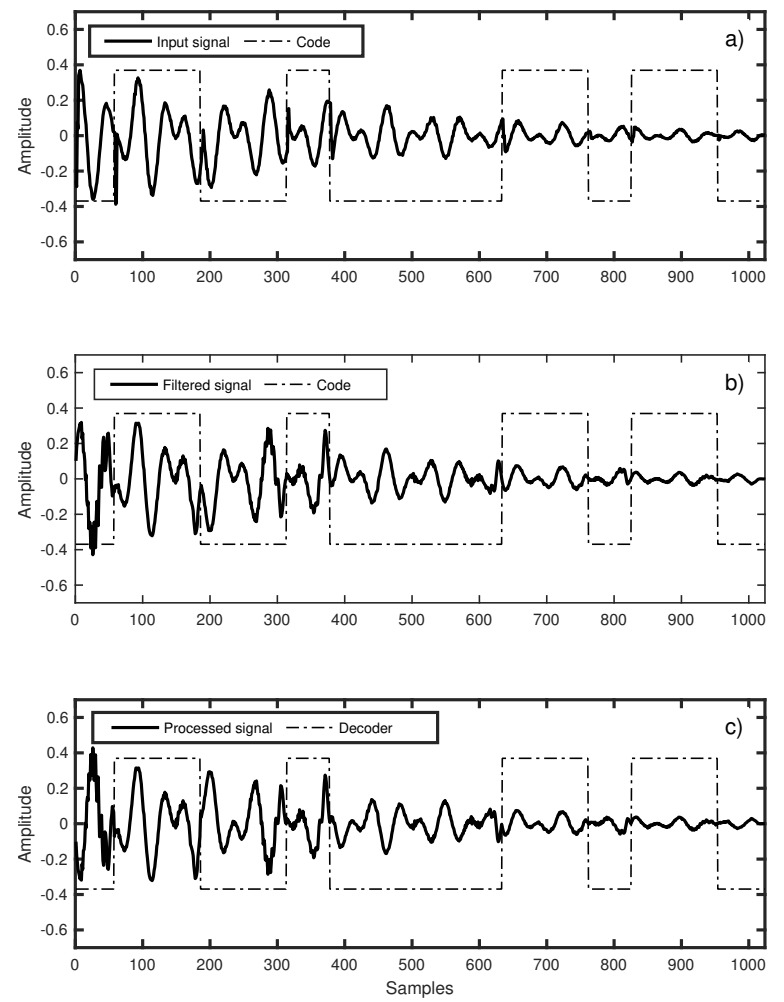

Fig. 13. Illustration of experiment. a) Collected (beat frequency) signal, b) filter output, and c) decoded signal. 


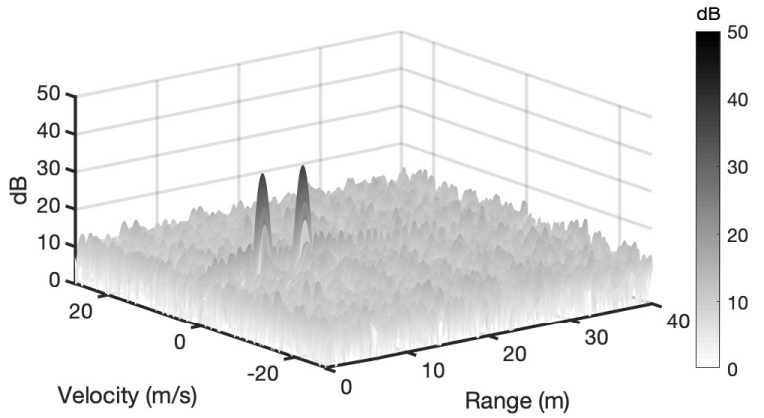

Fig. 14. Range Doppler plot of the experimental data after proposed processing.

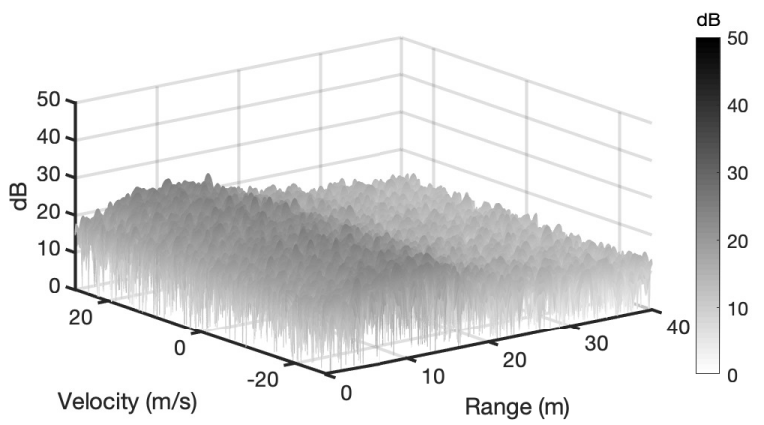

Fig. 15. Range Doppler plot of the experimental data without decoding.

Doppler processing is shown in Figure 14, where two stationary targets are clearly focused. To see the qualitative improvement, a range-Doppler plot of the experimental data without any decoding is illustrated in Figure 15. As seen from the figure, results obtained from the experimental data without any processing spread through the range dimension, due to the spread spectrum nature of the phase codes.

\section{Interference Mitigation On The PC-LFMCW RADAR}

As mentioned before, in a classical FMCW radar, spectral estimation techniques are used to estimate the range from the received beat signal, since target returns appear as constant frequency tones. One may achieve the target's range by applying the Fourier Transform to the received beat signal (see Appendix A)

$$
x_{B}(t) \stackrel{\mathcal{F}}{\rightarrow} X_{B}(f) .
$$

When perfect decoding is applied as $C^{*}(t-\tau) x_{B}(t)$, the system response (26) converges to a traditional FMCW output (39), whose first null appears at $f= \pm 1 / T$ or in terms of range

$$
R_{\text {null,decoding }}=\frac{c}{2 k} f= \pm \frac{c}{2 k} \frac{1}{T}= \pm \frac{c}{2 B}
$$

Thus, the Rayleigh (null-to-null) resolution or compressed pulse length is $c / B$. If there is an interference with a different code or no code at all, the system response is not focused and spread through the range domain due to the coding term (38). In these cases, the first null appears at

$$
R_{\text {null }, w / \text { decoding }}=\frac{c}{2 k} f=\frac{c}{2 k} \pm \frac{1}{T_{c}}= \pm L_{c} \frac{c}{2 B} .
$$

Similarly, the Rayleigh resolution for the undecoded signal output is $L_{c} c / B$.

The pulse compression ratio (the pulse length before processing, compared to the pulse length after processing) or pulse bandwidth product for decoded signal is

$$
P C R_{\text {decoding }}=\frac{c T}{c / B}=B T,
$$

where as for undecoded (without any decoding) signal,

$$
P C R_{w / \text { decoding }}=\frac{c T}{L_{c} c / B}=\frac{B T}{L_{c}}=B T_{c} .
$$

The signal-to-noise ratio (SNR) after pulse compression compared to the SNR before pulse compression is equal to the time bandwidth product $(B T)$ of the signals (for real signals such as in FMCW radar two times the bandwidth product $2 B T$ [22]). Since the SNR is proportional to the time bandwidth product, the decoded signal to undecoded signal ratio, in other words the improvement in signal to interference (SIR) ratio can be written as

$$
\begin{aligned}
S I R & =\frac{S N R_{\text {with decoding }}}{S N R_{w / \text { decoding }}} \\
& =10 \log \left(\frac{B T}{B T_{c}}\right) \\
& =10 \log \left(L_{c}\right) .
\end{aligned}
$$

\section{A. Simulations}

To show the effectiveness of the proposed method under an interference as well as to demonstrate the improvement in SIR, we set up simulations and an experiment.

For both the simulation and the experimental setup, we choose Zero-correlation Zone (ZCZ) sequences [28]-[30], due to their good auto and cross-correlation properties (this also demonstrates the applicability of the proposed method with different code sequences). The construction of ZCZ sequences with a code length of 64 is done according to [30]. Figure 16 illustrates the normalized auto-correlation and cross-correlation of the two selected codes. The duration of each chip is set to $0.40 \mu \mathrm{s}$, so that 64-bit ZCZ code perfectly covers the ADC sampling interval (which is $25.6 \mu$ s or 1024 samples at 40 $\mathrm{MSa} / \mathrm{s}$ sampling).

The proposed PC-LFMCW radar system architecture is used together with group delay filters to correct the time delay of coded waveforms before decoding. The system response for a point target at zero range $\left(\tau=0\right.$, thus $\left.f_{b}=0\right)$ is illustrated in Figure 17 with and without decoding. The decoded signal makes a perfect sinc pattern with a first null position at $R_{\text {null,decoding }}= \pm 0.37 \mathrm{~m}$, whereas the undecoded signal, that consists of multiple sinc functions (26), makes its first zero-crossing (null) at $R_{\text {null,decoding }}= \pm 27.28 \mathrm{~m}$. As seen from the figure, unlike the traditional spread spectrum techniques, where the signal spreads in the frequency domain 


$$
X_{B}(f)=T_{c} \operatorname{sinc}\left(f T_{c}\right) e^{-j 2 \pi f \tau} \sum_{n=1}^{L_{c}} e^{j \phi_{n}} e^{-j 2 \pi f(n-1 / 2) T_{c}} \otimes \frac{T}{2}\left[\operatorname{sinc}\left(\left(f-f_{b}\right) T\right)+\operatorname{sinc}\left(\left(f+f_{b}\right) T\right)\right] e^{-j 2 \pi f(\tau+T / 2)}
$$
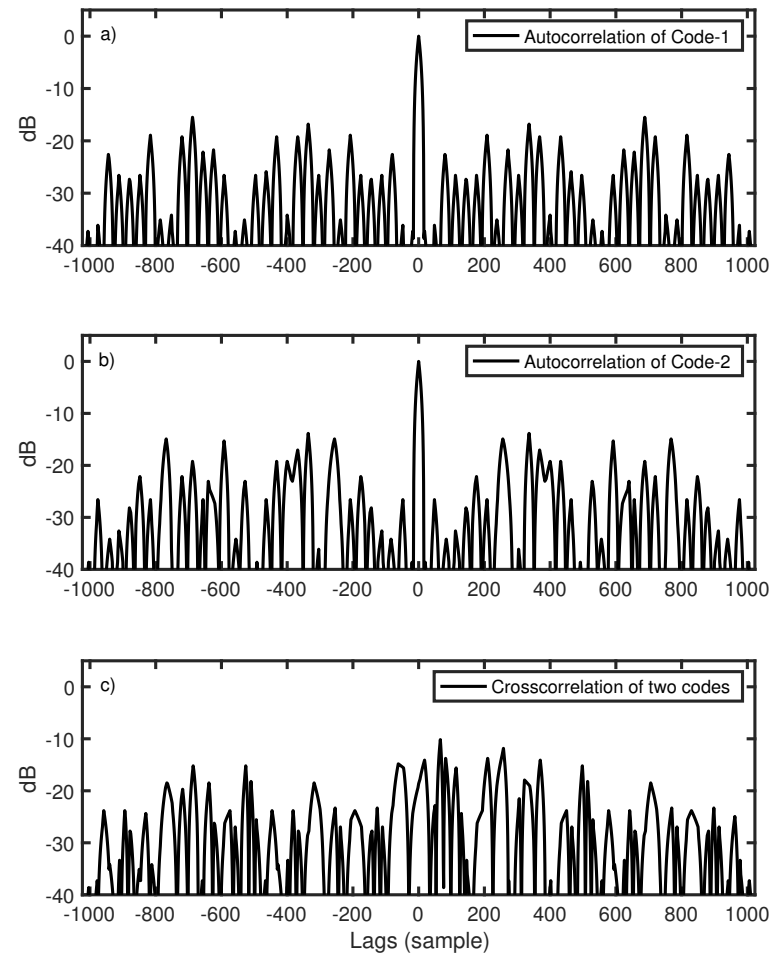

Fig. 16. Normalized auto-correlation and cross-correlation of two codes used for experiments

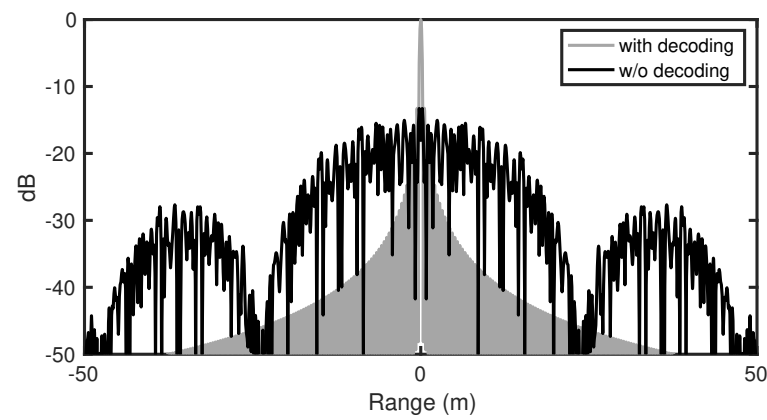

Fig. 17. Simulation of system response to a point target (zero-padded range processing). Decoded signal focused well, whereas undecoded signal spread in range dimension (beat frequency spectrum)

of the transmitted signal, in the proposed architecture the spreading factor is moved to the beat frequency spectrum (which is known as range domain). Due to the spreading, the peak of the interference signal has a lower energy, which is $10 \log (L c)=18.06 \mathrm{~dB}$ lower than the decoded signal.

\section{B. Experiments}

The simulated interference resilient system is also realized through an experimental facility that is explained in Section IV-C. In this experiment (which is the worse case interference scenario), two time-synchronous radar systems, separated
$5 \mathrm{~m}$ apart in range direction, share the same frequency spectrum (bandwidth and center frequency are same for each radar). Both transmit their own phase-coded LFM waveform. Each radar has its own ZCZ sequence (whose auto and crosscorrelation are illustrated in Figure 16). The collected signal is dechirped with a pure LFM waveform at each radar, to create the beat signal. Figure 18 shows the range-Doppler processing of the collected signal. As seen from the figure, two radars interfere with each other and their transmitted waveforms are mixed. As a result each radar sees a ghost target in addition to the actual target (even though there is only a single target). Moreover, the SNR of the actual target signature is also low due to the interference and non perfect envelope alignment. Figure 19 and Figure 20 show the range-Doppler processing for each radar, after the decoding with their own phase code by the proposed processing technique. As seen from the figure, due to the orthogonal code sequence, the ghost target in each radar is suppressed, which yields an increased SIR. Moreover, the perfect decoding helps to better focus the target signature so the SNR loss due to coding can be recovered.

These effects can be seen clearly in range profiles. Figure 21 shows a range profile of a single pulse for a collected signal without decoding and with decoding, by using two different code sequences. Since each pulse is coded individually, the interference mitigation, "10log $\left(L_{c}\right)$ SIR", can be observed at target locations in range profiles of a single pulse. The overall processing gain, after range-Doppler processing, can be seen in Figure 22. As seen from the figures, maximum interference mitigation occurs at the target's location, due to the used orthogonal codes sequences.

\section{Discussion AND Future WORK}

\section{A. Hardware Implementation}

A group delay filter that satisfies the requirements can be implemented into the hardware architecture to reduce the processing load and the discretization errors in the digital domain. The coded signal can be delayed and mixed with the filtered signal, to decode the beat frequencies before analog to digital conversion. A simplified block diagram of such an architecture is illustrated in Figure 23. The proposed hardware implementation is suitable for interference mitigation without any extra signal processing. A microcontroller can directly compute the 2D-FFT of the digitized output signal for rangeDoppler processing.

\section{B. Work in progress}

It should be noted that it is hard to generalize qualitative and quantitative results at this stage, because it depends on multiple factors, such as the transmited signal's parameters, code family and length, filter length etc. Since this paper aims to demonstrate the proof of concept, the optimization of such 


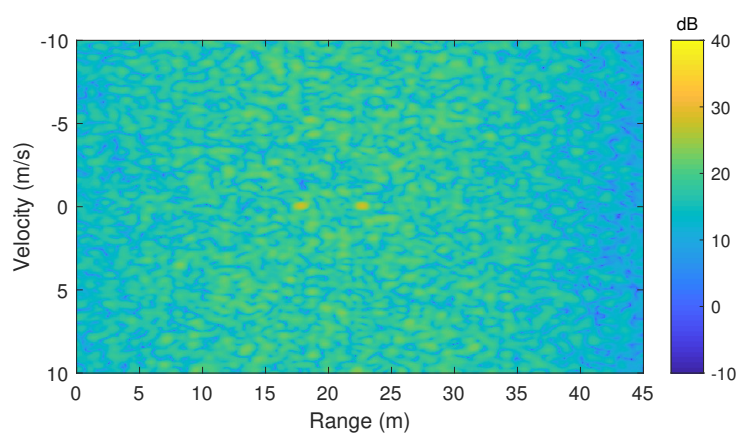

Fig. 18. Collected signal's range-Doppler output as seen from Radar-A and Radar-B which are apart from each other by $5 \mathrm{~m}$.

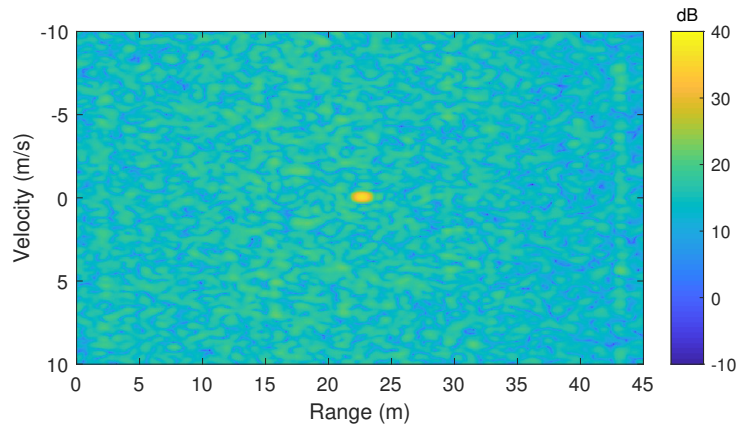

Fig. 19. Collected signal's range-Doppler output after proposed method as seen from Radar-A.

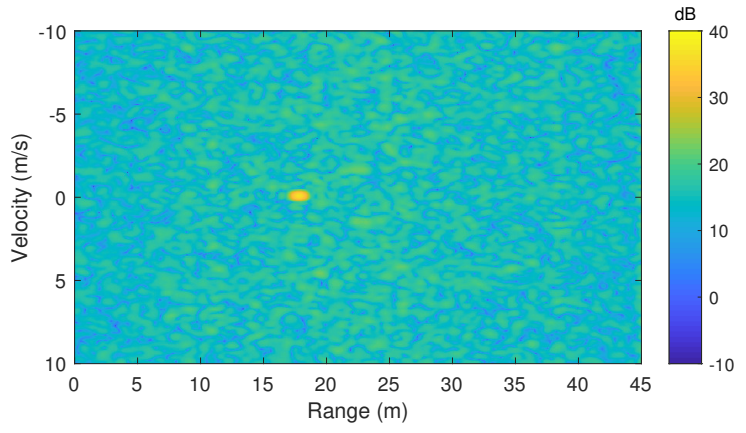

Fig. 20. Collected signal's range-Doppler output after proposed method as seen from Radar-B.

factors is not included in this paper, which is currently in progress. The investigation of full synchronous MIMO, as well as joint sensing and communication by using the proposed method, are left as ongoing research.

\section{CONCLUSION}

This paper proposes a phase-coded FMCW (PC-FMCW) radar system together with its appropriate signal processing, which reduces the sampling requirement of the received signals, by taking advantage of stretch processing while making the use of phase coding possible. Moreover, unlike the traditional phase-coded CW radar systems, the proposed system architecture does not need multiple correlators or an ADC with a large bandwidth in the hardware stage. Thus it reduces the architecture complexity compared to the approaches in literature.

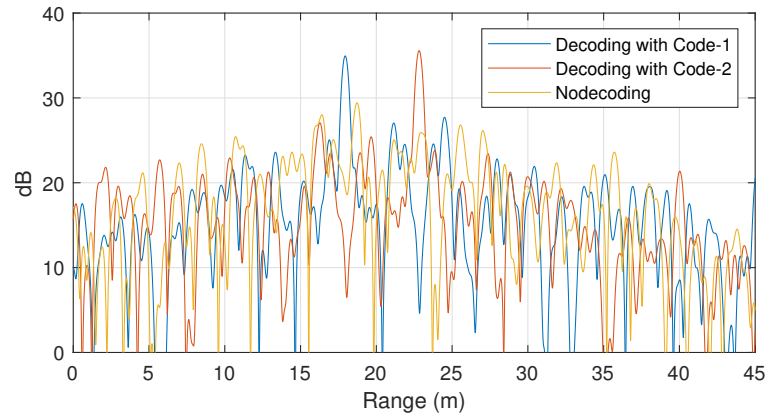

Fig. 21. Single pulse range processing.

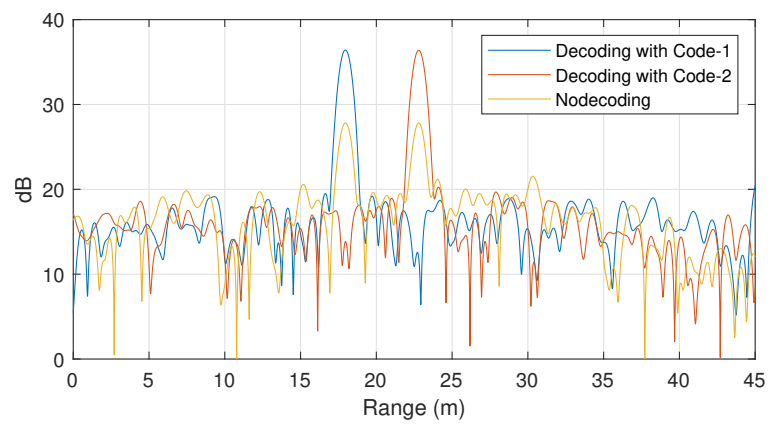

Fig. 22. Rrange-cut after range-Doppler processing.

We introduce a hybrid (software and hardware) system architecture, which transmits phase coded LFM waveforms and dechirps the received signals with a pure LFM waveform. This system architecture allows us to preserve phase coding through dechirp processing. After this, we propose to use a group delay filter to adjust the envelopes of beat frequencies, shifted due to propagation delay, and we derive an appropriate decoding signal. As a result, the LFM waveforms coded prior to transmission are successfully decoded in the FMCW radar receiver. Decoded waveforms are used to achieve range and velocity information of the targets. The success of the

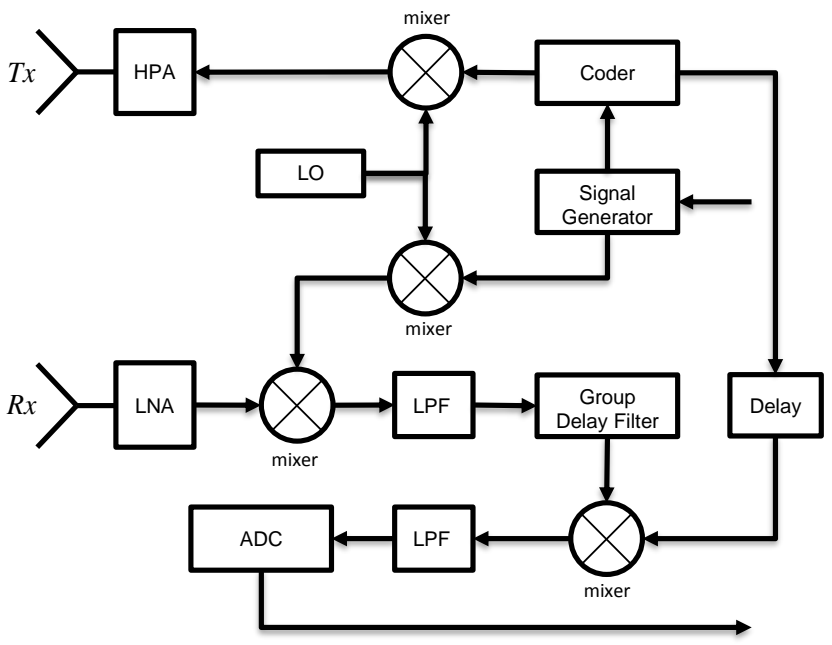

Fig. 23. Simplified block diagram of a proposed Phase-Coded FMCW radar system architecture. Envelop alignment and decoding are moved from digital domain to hardware domain. 
proposed PC-FMCW system is illustrated by simulations for an automotive radar scenario and verified by experiments.

We concentrate on the automotive radar interference mitigation problem and quantitatively and qualitatively show the improvement in SIR for interference mitigation. As demonstrated by simulations and experiments, the proposed method has a great potential to address the interference suppression problem for automotive radars in super-dense environments.

\section{ACKNOWLEDGMENT}

This project is funded through the TU Delft Industry Partnership Programme (TIPP) from NXP Semiconductors N.V and Holland High Tech Systems and Materials (TKIHTSM/18.0136) under the project "Coded-radar for Interference Suppression in Super-Dense Environments" (CRUISE).

\section{APPENDIX A}

\section{Fourier Transform of Phase Coded Beat Signal}

The received beat signal (11) can be split into two main parts by letting

$$
d(t)=\frac{C(t-\tau)}{2}
$$

and

$$
e(t)=\operatorname{rect}\left(\frac{t-\tau-T / 2}{T}\right) \cos \left(2 \pi f_{b} t+\phi_{c}\right) .
$$

Then the received beat signal (11) can be simplified to

$$
x_{B}(t)=d(t) e(t),
$$

which can be written equivalently in frequency domain by using the convolution property of Fourier transform as

$$
X_{B}(f)=D(f) \otimes E(f) .
$$

The Fourier Transform of the binary phase code $C(t)$ is given by [31],

$$
C(f)=P(f) \sum_{n=1}^{L_{c}} e^{j \phi_{n}} e^{-j 2 \pi f(n-1 / 2) T_{c}}
$$

where $P(f)$ is the Fourier transform of a single chip centered at $t=0$, which is

$$
P(f)=\frac{\sin \left(\pi f T_{c}\right)}{\pi f}=T_{c} \operatorname{sinc}\left(f T_{c}\right) .
$$

Then, the Fourier transform of a time delayed binary phase code $\mathcal{F}\{c(t-\tau)\}$ can be written as

$$
D(f)=T_{c} \operatorname{sinc}\left(f T_{c}\right) e^{-j 2 \pi f \tau} \sum_{n=1}^{L_{c}} e^{j \phi_{n}} e^{-j 2 \pi f(n-1 / 2) T_{c}} .
$$

The Fourier transform (33) can be written as

$$
\begin{array}{r}
E(f)=\frac{T}{2}\left[\operatorname{sinc}\left(\left(f-f_{b}\right) T\right)+\operatorname{sinc}\left(\left(f+f_{b}\right) T\right)\right] \\
e^{-j 2 \pi f(\tau+T / 2)} .
\end{array}
$$

Finally, Fourier transform of phase coded beat signals can be written as (26) by putting (38) and (39) into (35).

\section{REFERENCES}

[1] R. M. Davis, R. L. Fante, and R. P. Perry, "Phase-coded waveforms for radar," IEEE Transactions on Aerospace and Electronic Systems, vol. 43, no. 1, pp. 401-408, January 2007.

[2] F. Uysal and S. Sanka, "Mitigation of automotive radar interference," in 2018 IEEE Radar Conference (RadarConf18), April 2018, pp. 04050410.

[3] F. Uysal, "Synchronous and asynchronous radar interference mitigation," IEEE Access, vol. 7, pp. 5846-5852, 2019.

[4] Jian, MIMO radar signal processing. Hoboken, NJ: J. Wiley \& Sons, 2009.

[5] F. C. Robey, S. Coutts, D. Weikle, J. C. McHarg, and K. Cuomo, "MIMO radar theory and experimental results," in Conference Record of the Thirty-Eighth Asilomar Conference on Signals, Systems and Computers, 2004., vol. 1, Nov 2004, pp. 300-304 Vol.1.

[6] Z. Dunn, M. Yeary, F. Uysal, and C. Fulton, "Low sidelobe pseudoorthogonal code sets through particle swarm optimization," in 2016 IEEE Radar Conference (RadarConf), May 2016, pp. 1-4.

[7] B. Bhagavan, "Signal processor for binary phase coded CW radar." Computer Sciences Corp., Huntsville, AL, Tech. Rep., 1977.

[8] N. Levanon and B. Getz, "Comparison between linear fm and phasecoded CW radars," IEE Proceedings - Radar, Sonar and Navigation, vol. 141, no. 4, pp. 230-240, Aug 1994.

[9] C. Sahin, J. G. Metcalf, and S. D. Blunt, "Characterization of range sidelobe modulation arising from radar-embedded communications," in International Conference on Radar Systems (Radar 2017), Oct 2017, pp. 1-6.

[10] C. Sahin, J. Jakabosky, P. M. McCormick, J. G. Metcalf, and S. D. Blunt, "A novel approach for embedding communication symbols into physical radar waveforms," in 2017 IEEE Radar Conference (RadarConf), May 2017, pp. $1498-1503$.

[11] M. R. Franceschini and J. B. McKitterick, "FMCW radar with phase encoded data channel," Jan. 9 2018, uS Patent 9,864,043.

[12] J. J. M. de Wit, W. L. van Rossum, and A. J. de Jong, "Orthogonal waveforms for FMCW MIMO radar," in 2011 IEEE RadarCon (RADAR), May 2011, pp. 686-691.

[13] E. H. Kim and K. H. Kim, "Random phase code for automotive MIMO radars using combined frequency shift keying-linear FMCW waveform," IET Radar, Sonar Navigation, vol. 12, no. 10, pp. 1090-1095, 2018.

[14] R. Feger, H. Haderer, and A. Stelzer, "Optimization of codes and weighting functions for binary phase-coded FMCW MIMO radars," in 2016 IEEE MTT-S International Conference on Microwaves for Intelligent Mobility (ICMIM), May 2016, pp. 1-4.

[15] J. Reneau and R. R. Adhami, "Phase-coded LFMCW waveform analysis for short range measurement applications," in 2014 IEEE Aerospace Conference, March 2014, pp. 1-6.

[16] J. Reneau and R. Adhami, "Design of a short range continuous wave compound phase coded linear frequency modulation radar sensor," Progress In Electromagnetics Research, vol. 82, pp. 115-135, 2018.

[17] D. M. Hemmingsen, P. M. McCormick, S. D. Blunt, C. Allen, A. Martone, K. Sherbondy, and D. Wikner, "Waveform-diverse stretch processing," in 2018 IEEE Radar Conference (RadarConf18), April 2018, pp. $0963-0968$

[18] P. M. McCormick, C. Shain, S. D. Blunt, and J. G. Metcalf, "FMCW Implementation of phase-attached radar-communications (PARC)," in 2019 IEEE Radar Conference (RadarConf19), April 2019.

[19] D. Barrick, "FM/CW Radar Signals and Digital Processing," NOAA Environmental Research Laboratories, Boulder, Colorado, Tech. Rep., 1973.

[20] J. Tierney, C. Rader, and B. Gold, "A digital frequency synthesizer," IEEE Transactions on Audio and Electroacoustics, vol. 19, no. 1, pp. 48-57, March 1971.

[21] NXP Semiconductors, TEF810X Fully-Integrated $77 \mathrm{GHz}$ Radar Transceiver, www.nxp.com, 2019 (accessed January 20, 2019). [Online]. Available: https://www.nxp.com/products/rf/radar-transceivers/ tef810x-fully-integrated-77-ghz-radar-transceiver:TEF810X

[22] M. Jankiraman, FMCW radar design. Norwood, MA: Artech House, 2018.

[23] S. Engelberg, Digital signal processing : An experimental approach. Berlin London: Springer, 2008.

[24] A. Antoniou, Digital Signal Processing: Signals,Systems and Filters. McGraw-Hill NewYork, 2006.

[25] T. Kasami, "Weight distribution formula for some classes of cyclic codes. coordinated science lab., univ," Urbana Tech Rep, pp. 1966-04, 1966. 
[26] J. Overdevest, F. Jansen, F. Uysal, and A. Yarovoy, "Doppler Influence on Waveform Orthogonality in 79GHz MIMO Phase-Coded Automotive Radar," IEEE Transactions on Vehicular Technology, 2019.

[27] M. A. Richards, Fundamentals of radar signal processing. Tata McGraw-Hill Education, 2005.

[28] P. Z. Fan, N. Suehiro, N. Kuroyanagi, and X. M. Deng, "Class of binary sequences with zero correlation zone," Electronics Letters, vol. 35 , no. 10, pp. 777-779, May 1999.
[29] H. Haderer, R. Feger, C. Pfeffer, and A. Stelzer, "Millimeter-wave phasecoded CW MIMO radar using zero- and low-correlation-zone sequence sets," IEEE Transactions on Microwave Theory and Techniques, vol. 64, no. 12, pp. 4312-4323, Dec 2016.

[30] B. Fassi, "A New Class of Binary Zero Correlation Zone Sequence Sets," IOSR Journal of Electronics and Communication Engineering (IOSRJECE), vol. 5, no. 3, pp. 15-19, 2013.

[31] M. A. Richards, Principles of modern radar. Raleigh, NC: SciTech Pub, 2010. 